Meta

Journal des traducteurs

Translators' Journal

\title{
De la phraséologie à la traductologie proactive : essai de synthèse des fondements théoriques sous-tendant la recherche en phraséologie
}

\section{Mojca Pecman}

Volume 50, numéro 4, décembre 2005

Pour une traductologie proactive - Actes

For a Proactive Translatology — Proceedings

Por una traductología proactiva - Actas

URI : https://id.erudit.org/iderudit/019853ar

DOI : https://doi.org/10.7202/019853ar

Aller au sommaire du numéro

Éditeur(s)

Les Presses de l'Université de Montréal

ISSN

0026-0452 (imprimé)

1492-1421 (numérique)

Découvrir la revue

Citer cet article

Pecman, M. (2005). De la phraséologie à la traductologie proactive : essai de synthèse des fondements théoriques sous-tendant la recherche en phraséologie. Meta, 50(4). https://doi.org/10.7202/019853ar
Résumé de l'article

Le présent article a pour but d'évaluer l'apport possible de la phraséologie à la traductologie. A l'heure actuelle, malgré le nombre croissant de travaux consacrés à la phraséologie, la plupart des découvertes ne semblent pas encore avoir trouvé d'écho dans le domaine de la traduction, notamment sur le plan théorique général. En effet, la phraséologie est souvent présentée davantage comme un domaine applicatif que comme un domaine de recherche fondamentale. Cet article montre toutefois que non seulement la phraséologie est en mesure d'apporter des réponses à certains problèmes de traduction, mais aussi qu'elle doit cette capacité aux fondements mêmes qui sous-tendent toute approche phraséologique de la langue. L'article présente ainsi une vision d'ensemble des postulats sur lesquels repose la recherche en phraséologie en revisitant certains concepts fondamentaux et communs à ces deux disciplines. 


\title{
De la phraséologie à la traductologie proactive : essai de synthèse des fondements théoriques sous-tendant la recherche en phraséologie
}

\author{
Mojca Pecman \\ Université de Nice - Sophia Antipolis, Nice, France \\ pecman@unice.fr
}

\begin{abstract}
RESUME
Le présent article a pour but d'évaluer l'apport possible de la phraséologie à la traductologie. A l'heure actuelle, malgré le nombre croissant de travaux consacrés à la phraséologie, la plupart des découvertes ne semblent pas encore avoir trouvé d'écho dans le domaine de la traduction, notamment sur le plan théorique général. En effet, la phraséologie est souvent présentée davantage comme un domaine applicatif que comme un domaine de recherche fondamentale. Cet article montre toutefois que non seulement la phraséologie est en mesure d'apporter des réponses à certains problèmes de traduction, mais aussi qu'elle doit cette capacité aux fondements mêmes qui sous-tendent toute approche phraséologique de la langue. L'article présente ainsi une vision d'ensemble des postulats sur lesquels repose la recherche en phraséologie en revisitant certains concepts fondamentaux et communs à ces deux disciplines.
\end{abstract}

\begin{abstract}
The aim of the article is to evaluate to what extent the recent advances in phraseology may contribute positively to the research in traductology. Despite the significant increase of research works devoted to the field of phraseology, up until now most of the findings do not seem to have found their use in the domain of traductology, specifically from a theoretical point of view. Phraseology is indeed often presented as a domain of applied research rather than of fundamental research. The article shows that nevertheless phraseology is not only capable of resolving some specific problems of translation but that it owes this capacity to its underlying assumptions which stand behind every phraseological approach of language. The article thus offers an overall view of theoretical premises in phraseology by examining a number of fundamental concepts common to both disciplines, phraseology and traductology.
\end{abstract}

\section{MOTS-CLES/KEYWORDS}

phraséologie, traduction, théorie linguistique, recherche fondamentale, phraseology, translation, linguistic theory, fundamental research

\section{Introduction}

Depuis Vinay et Darbelnet (1977) la linguistique contrastive a fait du chemin, notamment en intégrant les divers phénomènes de collocabilité ${ }^{1}$ dans la problématique du transfert de sens entre diverses langues. L'importance de la collocabilité dans le passage d'une langue à l'autre constitue certes un fait reconnu de longue date, mais ce n'est que depuis les années quatre-vingts qu'il reçoit toute l'attention qui lui est due. La conséquence directe de ce regain d'intérêt est la naissance d'un nouveau domaine de recherche : la phraséologie. Dès lors, une question se pose : la recherche en phraséologie est-elle en mesure de répondre aux besoins de la traduction de demain ? A l'heure 
actuelle, malgré le nombre croissant de travaux consacrés à la phraséologie (cf. Sinclair (1991), Moon (1998), Mel' uk et al. (1995 \& 1999), Gross (1996), etc.), la plupart des découvertes ne semblent pas encore avoir trouvé d'écho dans le domaine de la traduction, notamment sur le plan théorique général. La phraséologie est en effet souvent présentée davantage comme un domaine applicatif que comme un domaine de recherche fondamentale, même si dans son rôle applicatif elle est aussi parfois sujette au scepticisme. Aussi, les fameuses « unités de traduction » dont parlent déjà Vinay et Darbelnet (1977 : 36-43) et qui sont au cœur des recherches en phraséologie, ne sont aux yeux de Ladmiral (1994: 211) « qu'un auxiliaire pédagogique approximatif ».

Si l'on examine cependant les postulats sur lesquels repose la recherche en phraséologie (cf. Pecman, 2004 : 57-68), il devient évident que l'approche phraséologique de la langue implique des fondements théoriques solides. La plupart de ces postulats linguistiques sont d'ailleurs connus depuis de nombreuses années et partagés par la communauté linguistique internationale. Leur conjonction mutuelle offre, à notre avis, un nouvel éclairage sur la problématique de la construction du sens en langue. En cela, la recherche en phraséologie s'inscrit tout naturellement dans les objectifs d'une traductologie proactive. Le présent article se propose ainsi d'explorer les bases théoriques de la phraséologie à la lumière des préoccupations propres à la traductologie, en revisitant certains concepts fondamentaux et communs à ces deux disciplines : les concepts de combinatoire, d'idiomaticité, d'économie, de créativité, de connaissance ou encore d'interaction sociale. Nous n'omettrons pas de mentionner également les limites de l'analyse phraséologique de la langue.

\section{Mécanisme de restriction combinatoire comme processus fondamental d'idiomatisation}

La phraséologie repose sur le phénomène de restriction combinatoire : par exemple on acceptera des suites comme to sharpen a pencil ou tailler un crayon; cependant, alors que to sharpen a knife est acceptable pour un anglophone, pour un francophone *tailler un couteau ne l'est pas puisque les règles de la combinatoire lexicale imposent l'expression aiguiser un couteau. En croate, par exemple, on a affaire à un hapax : šiljiti olovku, puisque le verbe šiljiti, dérivé du nom de l'instrument ( šiljilo « taille crayon »), ne connaît que cet emploi-là dans la langue. Dès lors, il devient évident que le phénomène de restriction combinatoire s'apparente à la problématique de la construction du sens en langue, problématique au cœur d'une science comme la sémantique mais aussi, et surtout, comme la traductologie ou encore la phraséologie. Chaque élément d'une langue (tailler, sharpen, šiljiti, crayon, pen, olovka...) a son propre paradigme de valences dans cette langue. Une action spécifique $X$, en l'occurrence « couper un objet avec un instrument tranchant afin de lui donner une forme déterminée », sur un objet spécifique $\mathrm{Y}$, ici « bâtonnet de bois renfermant une mine de graphite servant à écrire », s'actualise spécifiquement, et souvent lexicalement d'une manière différente, dans diverses langues. Tailler, sharpen et šiljiti ont un sens identique dans le contexte des arguments crayon, pen et olovka. Auprès d'autres arguments éventuels, ils peuvent revêtir d'autres sens. Il s'agit là pour tout système linguistique de définir la valeur spécifique de chacun de ces éléments, valeur qui le distingue de tous les autres éléments entrant dans la composition du même système : si l'on pouvait combiner les unités lexicales à volonté, elles perdraient toutes leurs capacités différentielles. On revient ainsi, force nous est de l'admettre, à Saussure (1995 : 163) selon qui « la partie conceptuelle de la valeur [d'un terme] est constituée uniquement par des rapports et des différences avec les autres termes de la langue. »

L'essence même d'une analyse phraséologique de la langue est l'établissement des valences des unités lexicales à l'intérieur des langues particulières ou au regard des différentes langues. Cette démarche offre par conséquent des moyens méthodologiques pertinents pour observer la construction du sens à travers les langues (cf. extraction des unités polylexicales à partir de corpus 
parallèles ou comparables, construction de paradigmes combinatoires...), et par-là les moyens adéquats pour aider à son transfert (cf. mise à disposition de ressources lexicales sous forme de bases de données phraséologiques bilingues). La méthode la plus aboutie à ce jour de la prise en compte de la combinatoire lexicale pour la détermination du sens des unités lexicales est sans doute celle élaborée par Mel' uk (1995 \& 1999).

A l'origine du mécanisme de restriction combinatoire se trouve une propriété essentielle de la langue en tant que système : la propriété combinatoire. La langue constitue en effet un système combinatoire. Comme tout système de ce type, elle comporte d'un côté des éléments et, de l'autre, les règles qui régissent les relations qu'entretiennent ces éléments à l'intérieur du système. La particularité des systèmes combinatoires est de permettre, à partir d'un nombre limité d'éléments, la génération d'un nombre illimité d'objets finis. Pinker (1999:7) explique cette propriété des langues naturelles en précisant la conséquence d'une telle organisation systémique (i.e. le caractère exponentiel de la langue) : "Grammar is an example of a combinatorial system, in which a small inventory of elements can be assembled by rules into an immense set of distinct objects.

Combinatorial systems obey what Miller (1991) calls the Exponential Principle: The number of possible combinations grows exponentially (geometrically) with the size of the combination. Combinatory system can generate inconceivably vast numbers of products."

Là où la situation se complexifie, c'est que la combinatoire en langue, premièrement, n'est pas libre mais régie et, deuxièmement, n'est que partiellement régie par des règles qui relèveraient d'une science exacte, au moins en ce qui concerne le niveau lexical de la langue. Une grande partie de la combinatoire lexicale repose en effet non pas sur des règles syntactico-sémantiques (par exemple, de telles règles permettent de combiner facilement et correctement une unité lexicale comme froid avec des adjectifs dénotant un certain degré de sensation thermique désignée par cette unité : un grand froid, un froid polaire, un froid glacial... ou avec un verbe de mouvement : le froid arrive, le froid entre, le froid pénètre... ; ou encore de combiner une unité lexicale comme pomme avec des unités dénotant une action en relation avec ce fruit: manger une pomme, éplucher une pomme, laver une pomme, cueillir les pommes..., une qualité du fruit : pomme mûre, pomme rouge, pomme verte, pomme acidulée... ou encore une préparation à base de ce fruit : compote de pommes, beignet de pommes, chausson aux pommes, gâteau de pommes, tarte aux pommes...), mais sur des paramètres aussi complexes et variés que les paramètres sociaux, économiques, historiques, mythiques, imaginaires, culturels, etc. (par exemple, l'unité lexicale froid entrera ainsi en combinaison avec d'autres unités de la langue plus inattendues, comme canard dans l'expression un froid de canard; l'unité lexicale pomme quant à elle donnera des suites comme pomme de discorde, pomme d'Adam, la pomme de Newton, pomme empoisonnée, ou encore tomber dans les pommes). C'est ce qui pousse Pinker (1999: ix-x) à qualifier le langage de « mixture of mathematical beauty and human quirkiness ». En d'autres termes, la combinatoire lexicale est régie par deux principes opposés : le principe de liberté et le principe de contrainte.

\section{Double dynamique : liberté et contrainte combinatoires}

La combinatoire lexicale obéit à deux principes régisseurs qui s'opposent : un principe qui permet de combiner à volonté les éléments du système, pourvu qu'on applique les règles qui régissent les rapports entre ces éléments (i.e. règles qui assurent la grammaticalité de la construction), et un autre qui limite cette liberté combinatoire sous la pression de nombreux paramètres intra- et extralinguistiques. Un des premiers à avoir posé l'existence de ces deux principes opposés dans les langues est Jespersen (1992 : 14-22, cf. le titre de son chapitre : Expressions figées et expressions libres). Il est important toutefois de rappeler que la distinction entre la liberté combinatoire et le figement, comme le souligne d'ailleurs Jespersen (ibid. : 17), est une caractéristique non pas du seul niveau lexical mais de la langue en tant qu'ensemble. 
En effet, à tous les niveaux d'analyse linguistique, on rencontre des contraintes et des libertés. On peut déjà déceler cette double dynamique au niveau mérismatique, avec des traits distinctifs qui se combinent sans difficulté (par ex. en français les traits occlusif et dental comme dans des sons [t] et [d]) et ceux qui ne se combinent jamais (par ex. en français les traits constrictif et dental $)^{2}$. De même, au niveau phonématique, certaines combinaisons de phonèmes sont permises (par ex. en français $/ \mathrm{s} / \wedge / \mathrm{t} / \wedge / \mathrm{r} /, / \mathrm{s} / \wedge / \mathrm{k} / \wedge / \mathrm{r} /, / \mathrm{k} / \wedge / \mathrm{s} / \wedge / \mathrm{p} / \ldots$ ) alors que d'autres sont interdites (par ex. en français $* / \mathrm{s} / \wedge / \mathrm{r} / \wedge / \mathrm{k} /, * / \mathrm{p} / \wedge / \mathrm{r} / \wedge / \mathrm{t} /, * / \mathrm{p} / \wedge / \mathrm{r} / \wedge / \mathrm{s} / \wedge / \mathrm{t} / \ldots)^{3}$. Il en va de même avec le niveau morphématique où certaines associations de morphèmes sont courantes (par ex. question $n^{\wedge}(n) e r$, question $^{\wedge}(n) e m e n t$, question $n^{\wedge}(n)$ aire...) et d'autres n'auront jamais lieu (par ex. *question ${ }^{\wedge}(n)$ ier, ${ }^{*}$ question $^{\wedge}(n)^{\wedge}$ ière, ${ }^{*}$ question ${ }^{\wedge}(n)$ isme...). Au niveau des unités lexicales, on observe un phénomène semblable : certains mots se combinent volontiers (de $e^{\wedge}$ toute $e^{\wedge}$ façon, de $e^{\wedge}$ toute ${ }^{\wedge}$ manière, selon $^{\wedge}$ toute $^{\wedge}$ apparence) et d'autres pas (par ex. ${ }^{*}$ de $e^{\wedge}$ toute ${ }^{\wedge}$ apparence, ${ }^{*}$ selon $^{\wedge}$ toute $e^{\wedge}$ façon, *selon toute $^{\wedge}$ manière).

Plus tard, Sinclair (1991 : 109-110) reprend la dichotomie jespersienne en expliquant davantage son rôle dans la construction du sens. Les deux principes qui régissent la combinatoire lexicale, que Sinclair désigne par « le principe du libre choix » (open-choice principle) et «le principe de l'idiomaticité » (idiom principle), permettent selon lui d'expliquer comment le sens se dégage du texte. En effet, l'ambiguiité sémantique d'une unité lexicale souvent disparait si l'on se réfère au contexte. De façon semblable, Wray \& Perkins (2000 : 11) écrivent qu'une grande partie du processus communicatif repose sur un va-et-vient entre les processus créatifs et les processus holistiques, chacun ayant ses propres avantages : "The advantage of the creative system is the freedom to produce or decode the unexpected. The advantage of the holistic system is economy of effort ${ }^{4}$ when dealing with the expected."

\section{Economie de l'effort}

Outre ce contrôle de la combinatoire lexicale, la phraséologie s'inscrit effectivement dans la tendance générale de la langue à l'économie de l'effort : dès que le premier élément collocatif est prononcé ou lu, une série d'arguments possibles se mobilisent dans l'esprit de l'auditeur ou du lecteur, facilitant la compréhension par l'anticipation. Le traitement du phraséolexique peut ainsi être expliqué à l'aide du modèle de la Cohorte (Marslen-Wilson \& Welsh, 1978) selon lequel un auditeur sélectionne à l'aide d'un ou plusieurs phonèmes initiaux une cohorte de candidats commençant par cette même séquence acoustique, un mot étant reconnu lorsqu'il est le seul membre restant dans la cohorte (par exemple, en anglais la séquence [' $\left.\varepsilon \lambda{ }^{\prime}\right]$ active la cohorte de mots elevate, element et elephant mais dès que l'on a [' $\left.\varepsilon \lambda^{\prime} \phi\right]$, le mot peut être reconnu comme étant elephant).

Le principe de l'économie de l'effort consiste à maintenir, au cours de l'évolution des langues, un certain équilibre entre l'énergie dépensée et l'information transmise. C'est Martinet (2003 : 176-196) qui a mis le premier en évidence cette propriété des langues. Elle permet d'expliquer une série de phénomènes qui se produisent dans le langage et qui sont de nature variée. Par exemple, pourquoi le schwa chute-t-il dans certains contextes (ex. semaine prononcé [smEn], quatre livres prononcé [katliv"]) ou encore pourquoi est-on est passé d'une unité lexicale longue comme cinématographe aux unités cinéma puis ciné, ou encore d'une expression $X$ nous communique depuis Londres à $X$, depuis Londres?

L'acte de parole au niveau phraséologique de la langue est ainsi également sous-tendu par cette tendance naturelle de la langue à l'économie de l'effort dans la mesure où une unité phraséologique offre le moyen le plus rapide, le plus efficace et le plus percutant pour exprimer une idée ('accomplir une tâche en utilisant uniquement ce qui se trouve disponible sur place' $\rightarrow$ faire qch avec les moyens $d u$ bord ou 'parler à nouveau du sujet qui a été traité précédemment et qui constitue le propos de la discussion' $\rightarrow$ revenir à ses moutons), tant du point de vue de la 
production de la parole, c'est-à-dire du point de vue de l'émetteur, que du point de vue de la compréhension de la parole, c'est-à-dire du point de vue du récepteur. En effet, contrairement à ce qui se passe avec des locuteurs non natifs, du côté des locuteurs natifs l'utilisation d'une expression toute faite implique moins d'efforts que l'encodage ou le décodage d'une construction originale pour exprimer la même idée.

Un des premiers à avoir souligné la relation entre l'économie de la langue et le langage préconstruit, est Sinclair (1991 : 110) : "The principle of idiom is that a language user has available to him or her a large number of semi-preconstructed phrases that constitute single choices, even though they might appear to be analysable into segments. To some extent, this may reflect the recurrence of similar situations in human affairs; it may illustrate a natural tendency to economy of effort $t^{5}$; or it may be motivated in part by the exigencies of real-time conversation."

\section{Le rôle de la phraséologie dans la créativité langagière}

$\mathrm{Si}$, en raison de leur caractère préconstruit, les unités phraséologiques sont souvent placées à l'extrême opposé de la créativité langagière, il ne faut pas perdre de vue que toute créativité naît d'un écart par rapport à un schéma établi, et ne peut exister que du fait de l'existence de celui-ci. A toute atypie préexiste un type. C'est ce qui confère précisément à toute manifestation de créativité sa singularité, voire son étrangeté.

Aussi, si la phraséologie constitue un système holistique, il est tout à fait possible de procéder aux modifications des combinaisons lexicales récurrentes pour des raisons diverses mais qui relèvent toutes de la recherche d'un effet de sens particulier. C'est ce qu'on trouve par exemple dans cette phrase de Pinker (1999 : 91), « verbs of feather change together » qui fait écho à l'expression birds of feather flock together, connue de tous les anglophones. La délexicalisation est très souvent mise à contribution dans le discours journalistique, les slogans publicitaires ou encore la presse scientifique de vulgarisation avec le but de capter l'attention du lecteur (ex. Hextril enlève les maux de votre bouche. ; Dim : c'est mâle, c'est bien. ; L'argent fait le bonheur. ; L'appétit vient en mangeant, la pizza vient en l'appelant., L'ABC de la graphologie, Au cour de la comète, How to give science a bad name...).

Il s'agit, pour reprendre les termes de Gross (1996 : 19-20), de jeux de langage qui consistent à briser le carcan caractéristique des suites figées. En phraséologie, on parle alors du « défigement» (Gross, 1996), de « contre-création » (Hausmann, 1984) ou tout simplement de « variation stylistique ». Par ces jeux de mots créés à partir de déformations d'expressions connues, les unités phraséologiques participent à la fonction poétique du langage.

La créativité langagière peut ainsi être abordée d'une façon plus aisée si l'on applique une approche phraséologique des faits de langue. Ainsi, avec la prise en compte des schémas sousjacents du langage, la phrase de Pinker : "verbs of feather change together », au lieu d'être traduite par *les verbes de même plumage changent ensemble, pourrait être plus convenablement traduite par les verbes qui se ressemblent se conjuguent ensemble.

\section{Phraséologie et la dimension culturelle du langage}

Un autre point fort de la phraséologie réside dans sa prise en compte systématique de la dimension culturelle du langage lors de la mise en équivalence des unités phraséologiques. En effet, l'intérêt primordial accordé à la combinaison des unités lexicales propulse la phraséologie au rang des domaines qui cherchent à identifier les diverses marques d'ordre culturel dans le langage (cf. la parémiologie, la sociolinguistique, la psycholinguistique...). 
En cherchant à construire les unités de traduction, la phraséologie s'efforce de mettre en équivalence de la façon la plus adéquate possible les unités polylexicales qui reflètent une réalité culturelle.

Il peut s'agir simplement d'unités qui reflètent un fonds culturel commun à diverses langues sous une forme linguistique sensiblement identique. Les exemples suivants témoignent d'un fonds culturel partagé entre les civilisations anglophone et francophone, respectivement quant à leur passé historique, antique, mythique, biblique ou populaire : to cross the Rubicon = franchir le Rubicon, all roads lead to Rome $=$ tous les chemins mènent à Rome, Achilles heel $=$ talon d'Achille, séparer le bon grain de l'ivraie = to separate the wheat from the chaff, don't look a gift horse in the mouth = à cheval donné on ne regarde pas les dents.

Mais, une approche phraséologique de la langue permettra également d'apparier les unités sémantiquement apparentées qui se réalisent sous une forme linguistique plus ou moins différente : a labour of Hercules $=$ un travail de titan, until kingdom come = jusqu'à la fin des temps, to send $s b$ to Coventry = mettre qn en quarantaine, to work like a Trojan = travailler comme un forçat, beware of Greeks bearing gifts = ne faites jamais confiance à un ennemi; ou bien sous une forme linguistique foncièrement différente : to fiddle while Rome burns = se ficher de qch comme de l'an 40, great oaks from little acorns grow = les petits ruisseaux font les grandes rivières, pigs might fly = quand les poules auront des dents.

De même, elle tentera de mettre en relation les unités qui désignent des expériences spécifiques à une culture et qui se refusent à toute traduction facile : Bob's your uncle! = C'est simple comme bonjour! ; to be as safe as the Bank of England = être à toute épreuve ; Catch-22 situation = situation paradoxale, cercle vicieux; If music be the food of love, play on. = Si la musique est la nourriture de l'amour, joue encore.

C'est la raison pour laquelle la phraséologie est souvent considérée comme un terrain d'observation pour une approche anthropomorphique ou encore cognitive du langage, notamment par des phraséologues travaillant dans la tradition linguistique russe (cf. Teliya et al. 1998, Baranov \& Dobrovol'skij 1996).

\section{Phraséologie en tant qu'outil d'interaction sociale}

La phraséologie se révèle être également le produit et l'outil de l'interaction sociale. En ce sens, les unités phraséologiques permettent de mobiliser un fonds de valeurs partagées par une communauté linguistique donnée pour transmettre plus efficacement un message ou pour parvenir plus facilement à des fins d'ordre pragmatique. Par ce pouvoir d'interpellation du colocuteur, les unités phraséologiques participent à la fonction conative ou incitative du langage.

En ce sens, la recherche en phraséologie aborde toutes ces suites lexicales qui sont gelées par rapport à un contexte situationnel et que Mel' uk (1993 : 84) appelle les pragmatèmes (ex. ça fait un bail !, ça suffit comme ça!, quand on parle du loup...). Le repérage et la traduction systématiques de ces unités dans le cadre des projets de recherche en phraséologie bilingue devraient fournir des ressources lexicales utiles pour le traducteur (ça fait un bail ! = long time no see!, ça suffit comme ça! = enough's enough, quand on parle du loup = talk of the devil).

En effet, il existerait comme des schémas langagiers liés à des situations extralinguistiques. Ces schémas consisteraient à proposer, pour une situation donnée, un ou plusieurs choix pour agir verbalement selon un même cliché. Par exemple, pour dire que la réponse à la question posée est évidente, l'anglais propose les idiomes suivants (en guise de réponse du colocuteur à la question posée par le locuteur) : Is the Pope Catholic ? (litt. Le Pape est-il catholique ?), Do ducks swim ? (litt. Les canards savent-ils nager ?), etc. La langue croate offre pour le même scénario l'idiome Jeli more slano ? (litt. La mer est-elle salée ?). En français, ce type d'agissement linguistique n'existe pas mais on trouve le même phénomène par exemple face à un comportement langagier tel que celui 
qui se rencontre lorsqu'une personne éternue. Cette situation déclenche régulièrement un schéma cliché basé sur le dialogue : - À tes souhaits ! - Merci. ou - À tes souhaits ! - Merci. - À tes amours! - Que les tiennes durent toujours!.

Le point fort de la phraséologie est de se concentrer sur les unités de la langue en fonction de leurs conditions de production et leur rôle socio-pragmatique, et de tenter de mettre en rapport les équivalents possibles dans diverses cultures.

\section{Les limites de l'analyse phraséologique de la langue}

Si le but principal des recherches en phraséologie est de mettre en évidence les capacités combinatoires des unités lexicales, la difficulté majeure d'une analyse phraséologique de la langue (comme celle par exemple destinée à produire des ressources phraséologiques réutilisables, que ce soit sous forme d'unités phraséologiques monolingues, ex. frequently asked questions, ou sous forme d'unités de traduction, ex. frequently asked questions = foire aux questions) tient alors à notre impossibilité d'affirmer rigoureusement toutes les combinaisons acceptables entre les éléments composant le lexique d'une langue. Cette limite de l'analyse phraséologique touche notamment toutes ces combinaisons d'unités lexicales auxquelles de plus en plus de linguistes référent en termes de collocation ou de schéma collocationnel. La raison en est très simple : l'explicitation, voire la construction d'un répertoire de toutes les combinaisons possibles, et automatiquement de toutes celles qui sont interdites (i.e. des combinaisons restrictives), implique rien moins que la décomposition du mécanisme de l'économie de la langue, tel qu'il a été mis en évidence par Martinet (2003 : 168-169) et qui constitue le principe même du fonctionnement de la langue en tant que système exponentiel (cf. Pinker 1999 : 7). Gross et Clas (1997) ont essayé de contourner cet obstacle en forgeant la théorie de « classe d'objets » capable de rendre compte des paradigmes collocationnels non pas pour une seule unité lexicale (ex. démarrer la voiture) mais pour une série d'unités (ex. démarrer <transports routiers $>$ ).

Le deuxième obstacle auquel se heurtent les phraséologues travaillant dans la perspective bilingue est celui qui est très bien connu des traducteurs : il s'agit du clivage culturel. Les différences profondes entre diverses cultures rendent certaines unités phraséologiques intraduisibles. La phraséologie est uniquement en mesure de répertorier ces unités et de proposer des équivalents par paraphrase, comme dans cette expression croate : veži konja tamo gdje gazda

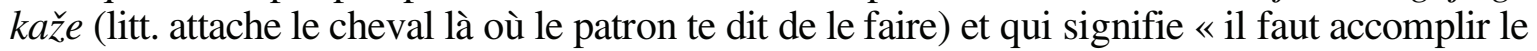
travail de manière à faire plaisir au patron même si on ne partage pas son avis » et que l'on pourrait traduire par : fait ce qu'on te dit de faire et ne pose pas de questions.

Enfin, en ce qui concerne le rôle des structures récurrentes dans la compréhension des créations linguistiques originales, il faut toutefois préciser que la créativité langagière dans son ensemble (notamment celle mise en œuvre dans le genre littéraire, et plus particulièrement encore dans la poésie), ne peut être expliquée qu'au regard d'une structure standard de la langue, comme le souligne très justement Baker (1998 : 5). Comme la traductologie, la phraséologie aura effectivement du mal à proposer des solutions miracles pour la traduction des séquences comme : Science sans conscience n'est que ruine de l'âme. ou Là tout n'est qu'ordre et beauté, luxe, calme et volupté.

\section{Conclusion}

Cette synthèse des fondements théoriques de la phraséologie montre que l'apport possible de la phraséologie à la traductologie est important. La phraséologie permet d'explorer un certain nombre de mécanismes qui gouvernent le fonctionnement des langues et qui sont souvent négligés dans l'étude de la langue et du passage d'une langue à l'autre car ils sont sentis comme un contrepoids à 
la cohérence de la grammaire ou encore comme un trait moindre par rapport à celle-ci (i.e. les mécanismes de restriction combinatoire, d'idiomatisation, d'économie de l'effort...). Ces mécanismes servent toutefois à accorder les éléments linguistiques de nature différente les uns par rapport aux autres ${ }^{6}$ afin d'assurer le fonctionnement harmonieux du système dans sa fonction d'expression d'une infinité de sens. Ils servent ainsi à ajuster le système global de la langue dans sa progression.

Certes, par rapport à la traductologie, la phraséologie constitue elle-même un champ de recherche assez récent (notamment dans un pays comme la France), et comme le souligne très justement Clas (1994 : 578), de surcroît démuni d'une théorie générale reconnue : «Si la constatation du phénomène de la collocation est quelque peu générale, de même que des relevés d'exemples sont nombreux, la théorisation, elle, reste beaucoup plus parcimonieuse et plus difficile à élaborer. »

Certes aussi, la phraséologie ne nous aidera pas à résoudre tous les problèmes de transfert du sens d'une langue à l'autre, notamment pas ceux dus aux différences profondes entre les cultures de divers peuples (reflétées par le vocabulaire et par la langue en général) dont parle si admirablement Mounin (1963), mais elle est tout à fait en mesure de nous fournir les moyens pour passer de la structure d'une langue à la structure d'une autre d'une façon moins hasardeuse et, par conséquent, de nous aider à faire passer un contenu, avec toute sa charge connotative et dénotative, dans une langue cible en accord avec la structure spécifique de celle-ci.

En effet, l'avantage de la phraséologie est de s'attaquer à ces endroits de la langue qui posent des problèmes lors du passage d'une langue à l'autre et pour lesquelles on peut encore espérer trouver des solutions. On ne trouvera probablement jamais une réponse qui éluciderait parfaitement le problème de traduction des unités désignant des réalités spécifiques à une civilisation (ex. pan bagnat ou scone) ou encore des unités désignant des réalités proches d'une façon spécifique (ex. poireauter « to hang about » ou kiss and fly « dépose minute »), mais n'est-il pas préférable que ce problème demeure à tout jamais ? En effet, si un jour il ne se posait plus à nous, cela voudrait dire que le monde entier est devenu uniforme et que toutes les richesses des civilisations se sont estompées au profit d'une réalité unique, voire d'une langue unique.

Avec cet article, nous avons voulu ainsi attirer l'attention sur l'importance de l'élaboration du volet théorique de la phraséologie et de la prise en compte de celui-ci par la théorie générale de la traductologie, comme prolégomènes à une réponse efficace aux besoins de la communication translinguisitique de demain. 


\section{NOTES}

1. Nous considérons que le phénomène de collocabilité opère à chaque fois que deux ou plusieurs unités lexicales manifestent une tendance, plus ou moins marquée, à être employées ensemble, peu importe la nature grammaticale des éléments entrant en combinaison ou de l'ensemble ainsi construit.

2. Ces traits se combinent cependant facilement en anglais où ils donnent lieu aux sons [ ] et [ð].

3. Pareillement, dans d'autres langues ces mêmes combinaisons pourraient être autorisées. C'est le cas par exemple en croate où les enchainements des phonèmes $/ \mathrm{s} / \wedge / \mathrm{r} / \wedge / \mathrm{k} /, / \mathrm{p} / \wedge / \mathrm{r} / \wedge / \mathrm{t} /$ et $/ \mathrm{p} / \wedge / \mathrm{r} / \wedge / \mathrm{s} / \wedge / \mathrm{t} /$ sont courants : ex. srkati (« siroter »), prtljaga («bagage ») et prst (« doigt»).

4. C'est nous qui soulignons.

5. C'est nous qui soulignons.

6. Par exemple, les morphèmes prépositionnels, tels que sur, en, dans, à, s'accordent par apport aux unités lexicales de nature nominale, tels que rue, route, voie, chemin, de la façon suivante: dans la rue, *sur la rue, *en (la) rue, à la rue, *dans la route, sur la route, en route, *à la route, *dans la voie, sur la voie, en voie, *à la voie, *dans le chemin, sur le chemin, en chemin, *au chemin. De manière sensiblement identique, des éléments linguistiques aussi divers que verbes, noms, adjectifs et prépositions s'accordent en adoptant les arrangements comme ceux que l'on trouve dans les séquences rentrer dans le droit chemin, trouver une difficulté sur son chemin, ne pas s'arrêter en si bon chemin, etc.

\section{REFERENCES}

BAKER, M. (1998) : «Réexplorer la langue de la traduction : une approche par corpus », in Meta 43-4, p. 1-7.

BARANOV, A. and DoBrovol'SKIJ, D. (1996): "Cognitive modeling of actual meaning in the field of phraseology", in Journal of Pragmatics 25-3, p. 409-429.

CLAS, A. (1994) : «Collocations et langues de spécialité », in Meta 39-4, p. 576-580.

GROSS, G. et CLAS (1997) : « Synonymie, polysémie et classes d'objets », in Meta 42-1, p. 147-154.

Gross, G. (1996) : Les expressions figées en français. Noms composés et autres locutions, coll. «L'essentiel français », Paris/Gap, Ophrys.

HAUSMANN, F. J. (1984): „Wortschatzlernen ist Kollokationslernen. Zum Lehren und Lernen französischer Wortverbindungen“, in Praxis des neusprachlichen Unterricht 31, p. 395-406.

JESPERSEN, O. (1992) : La philosophie de la grammaire (1924 pour l'éd. originale), coll. « Tel », Paris, Gallimard. LADMIRAL, J.-R. (1994) : Traduire : théorèmes pour la traduction, coll. « Tel », Paris, Gallimard.

MARSLEN-WILSON, W. and\& WELSH, A. (1978): "Processing interactions and lexical access during word recognition in continuous speech", in Cognitive Psychology 10, p. 29-63.

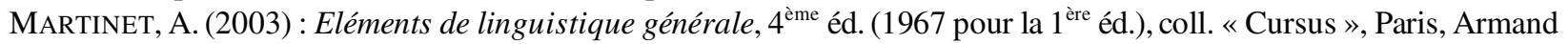
Colin.

MEL'CUK, I. (1993) : «La phraséologie et son rôle dans l'enseignement/apprentissage d'une langue étrangère », in

Etudes de Linguistique Appliquée 92, p. 82-113.

MEL'CUK, I., ClAS, A. et POLGUERE, A. (1995) : Introduction à la lexicologie explicative et combinatoire, Louvain-laNeuve, Duculot.

Mel'Cuk, I., Arbatchewsky-Jumarie, N., Iordanskaja L., Mantha, S. et Polguere, A. (1999) : Dictionnaire explicatif et combinatoire du français contemporain. Recherches lexico-sémantiques IV, Montréal, Les Presses de l'Université de Montréal.

MiLLER, G. (1991): The science of words, New York, W.H. Freeman.

Moon, R. (1998): Fixed Expressions and Idioms in English. A Corpus-based Approach, Oxford, Clarendon Press. Mounin, Georges (1963) : Les problèmes théoriques de la traduction, coll. « Tel », Paris, Gallimard.

PECMAN, M. (2004) : Phraséologie contrastive anglais-français : analyse et traitement en vue de l'aide à la rédaction scientifique, Thèse de doctorat, 9 déc. 2004, Dir. H. Zinglé, Université de Nice - Sophia Antipolis.

PINKER, S. (1999): Words and Rules. The ingredients of language, New York, Basic Books.

SAuSSURE, F. de (1995) : Cours de linguistique générale, $4^{\text {ème }}$ éd. (1916 pour la $1^{\text {ère }}$ éd.), Paris, Editions Payot.

SinClaIR, J. (1991): Corpus, Concordance, Collocation, Oxford, Oxford University Press. 
Teliya, V., Bragina, N., OpARINA, E. and SAndomirskayA, I. (1998): "Phraseology as a language of culture: its role in the representation of a cultural mentality", in COWIE, A. (Ed.) Phraseology: Theory, Analysis and Applications, Oxford, Oxford University Press, p. 55-75.

VinAy, J.-P. et DARBELNET, J. (1977) : Stylistique comparée du français et de l'anglais. Méthode de traduction, nouv. éd. revue et corr. (1958 pour la $1^{\text {ère }}$ éd.), Bibliothèque de stylistique comparée $\mathrm{n}^{\circ} \mathrm{I}$, Paris, Didier.

WrAY, A. and PERKINS, M. (2000): "The functions of formulaic language: an integrated model”, in Language \& Communication 20-1, p. 1-28. 